\title{
1 Comparative genomics of Staphylococcus capitis reveals determinants of
}

\section{2 speciation}

4 Authors:

5 Charlotte E. Chong ${ }^{1}$, Rebecca J. Bengtsson ${ }^{1} \&$ Malcolm J. Horsburgh ${ }^{1 *}$

6 Affiliations:

$7 \quad{ }^{1}$ Institute of Infection, Veterinary and Ecological Sciences, University of Liverpool, Merseyside,

8 United Kingdom.

9 Corresponding author:

10 M. J. Horsburgh

11 Institute of Infection, Veterinary and Ecological Sciences, University of Liverpool.

12 Email: M.J.Horsburgh@liverpool.ac.uk

13 Tel: +441517954569

$14 \quad$ Fax +441517954410

\section{Abstract}

Staphylococcus capitis is primarily described as a human skin commensal but is now emergent as an opportunistic pathogen isolated from bloodstream and prosthetic joint infections, and neonatal intensive care unit (NICU) associated sepsis. We used comparative genomic analyses of S. capitis to provide new insights of commensal scalp isolates from varying skin states, and to expand our current knowledge of the species populations (scalp isolates, $n=59$, contextual isolates, $n=127$ ). A highly recombinogenic population structure was revealed, with genomes including the presence of a range of previously described staphylococcal virulence factors, cell wall-associated proteins, and two-component systems. Genomic differences between the two described S. capitis subspecies were explored and reveal determinants associated exclusively with each. The subspecies ureolyticus was distinguished from subspecies capitis by differences in antimicrobial resistance genes and gene clusters linked to survival on the skin. This study will aid further 
research into classification of $S$. capitis and virulence linked phylogroups that is important to monitor the spread and evolution of S. capitis.

\section{$29 \quad$ Keywords}

Staphylococcus, capitis, genome, scalp, dandruff, genomics

\section{Introduction}

Staphylococcus capitis was first isolated from healthy human skin in 1975 and classified as a coagulase-negative Staphylococcus species (CoNS) (1). S. capitis is frequently found on the human scalp and the forehead, as it thrives in lipid-rich areas where sebaceous glands are abundant and was more recently associated with dandruff presenting scalps (1-4). S. capitis can be divided into two subspecies, capitis and ureolyticus, based on differences in their urease production and maltose fermentation (2). Current literature has additionally sought to characterise the two $S$. capitis subspecies isolated from NICUs, with respect to clinically relevant phenotypes such as- antimicrobial susceptibility, structure of the ica operon and biofilm formation $(5,6)$. Studies report increased prevalence of multidrug resistance (MDR), biofilm formation ability, and variation in the ica operon in S. capitis ssp. ureolyticus compared to $S$. capitis ssp. capitis (5), as well as differences in transcriptional response to erythromycin (6). Other published studies have also described the S. capitis subspecies though, none have sought to genotypically characterise them using WGS.

Most reports on S. capitis link it to a range of human diseases, being frequently isolated from prosthetic joint infections (7-9), prosthetic valve endocarditis (10-12) and late-onset sepsis in newborns at neonatal intensive care units $(5,13-16)$. The role of $S$. capitis in these infections was studied with reference to the well-described and clinically important species within the Epidermidis cluster group, i.e., Staphylococcus epidermidis and Staphylococcus caprae (17-19). S. capitis encodes important virulence factors required for biofilm production, persistence and immune evasion (17). S. capitis together with other CoNS species, such as S. epidermidis, are considered to have a lower virulence potential compared with the more virulent species $S$. aureus, since CoNS do not encode such an extensive suite of exotoxins associated with invasive infection $(20,21)$. 
Species of the CoNS possess a sufficient number of virulence factors to be classed as opportunistic pathogens, contributing significantly to nosocomial infection (22). Several CoNS possess a large repertoire of virulence factor genes including those linked to adhesion and biofilm formation, affording them both commensal and pathogenic traits $(20,22,23)$. CoNS are proposed to act as a reservoir for the uptake of mobile genetic elements. As commensals, they exist closely with other bacteria on skin and mucosal surfaces, and via recombination and frequent acquisition of mobile genetic elements they are able to increase their genetic diversity (23-25).

In this present study, we whole-genome sequenced 59 S. capitis isolates sampled from scalp skin and performed whole-genome sequencing analysis (WGSA), incorporating a further 127 publicly available sequences, with the aim to expand knowledge of $S$. capitis population structure, genotypic definition of subspecies and identify factors that are likely to contribute to virulence, competition, and colonisation.

Materials and Methods

\section{Dataset and bacterial isolates}

Whole-genome sequencing (WGS) was performed on 59 S. capitis isolates scalp skin, collected in the UK in 2017. Scalp samples were obtained using the method of Williamson and Kligman (26). The collection sample site was located and an appropriate clear and straight parting in the hair $\sim 10 \mathrm{~cm}$ long was secured to maximize exposure of the scalp. A Teflon cup $(18 \mathrm{~mm}$ internal diameter and $6 \mathrm{~cm}$ high) was placed onto the hair parting. A volume of $2.0 \mathrm{~mL}$ sampling collection medium (phosphate buffered saline plus $0.1 \%$ Triton-X-100) was applied to the collection site and the skin agitated in the liquid for one minute with a Teflon rod. The resulting $4.0 \mathrm{~mL}$ sample was transferred to a sterile tube. For each scalp sample taken, $100 \mu \mathrm{L}$ of wash was plated and when possible four distinct colonies were isolated from the following staphylococcal selective medium: (1\% (w/v) Tryptone (Oxoid), $0.5 \%(\mathrm{w} / \mathrm{v})$ lab lemco powder (Oxoid), $0.3 \%(w / v)$ yeast extract, $1.3 \%(w / v)$ agar (Lab M), $0.1 \%(w / v)$ sodium pyruvate (JT Baker Chemicals), 0.05\% (w/v) glycine (JT Baker Chemicals), 2.25\% (w/v) potassium 
lithium chloride (JT Baker Chemicals), $0.5 \%$ (v/v) glycerol (JT Baker Chemicals), 1.0\% (v/v) sodium azide (Sigma Aldrich), 3.0\% (v/v) sterile egg yolk emulsion (Lab M), pH 7.2).

Additionally, 7 skin isolates, including culture collection type strains represented both $S$. capitis subspecies were sequenced and included (ATCC 49325 (S. capitis subsp. ureolyticus) and ATCC 27840 (S. capitis subsp. capitis). Also included were published genomic data available from GenBank (https://www.ncbi.nlm.nih.gov/genbank/), Sequence Read Archive (https://www.ncbi.nlm.nih.gov/sra) or European Nucleotide Archive (https://www.ebi.ac.uk/ena), and accession numbers are listed in Supplementary Table 1 . All publically available $S$. capitis genomes were downloaded, subject to quality control, phylogenetically reconstructed (as described below) and Treemmer v0.3 was used to reduce redundancies within the public dataset, leaving 120 genomes to be included in further analyses (27).

\section{Genome sequencing and phylogenetic analysis}

All isolates selectively obtained in this study, together with 7 further strains comprising type strains and skin isolates, were grown in $10 \mathrm{~mL}$ BHI broth (Lab M) overnight, shaking at $37{ }^{\circ} \mathrm{C}$. Subsequently, $1 \mathrm{~mL}$ of each overnight culture was centrifuged for $1 \mathrm{~min}$ at $5000 \mathrm{rpm}$ and resuspended in $180 \mu 1$ lysis buffer (20 mM Tris- HCl pH8, 2 mM EDTA, 1.2\% Triton X-100); the cells of each clone were extracted to obtain high-quality genomic DNA using the QIAGEN DNeasy Blood \& Tissue Kit and eluted in $10 \mathrm{mM}$ Tris- $\mathrm{HCl}(\mathrm{pH} 8.5)$.

DNA concentration was measured using a Thermo Scientific Nanodrop, a Qubit plus visualisation after gel electrophoresis on $1 \%(\mathrm{w} / \mathrm{v}$ ) agarose gels (at $90 \mathrm{mV}$ for 40 min with a $1 \mathrm{~kb}$ ladder). For sequencing, the extracted DNA was required in a final volume of $60 \mu \mathrm{l}$ with a concentration between 1-30 ng $\quad \mu^{-1}$. Illumina DNA sequencing was performed by MicrobesNG (http://www.microbesng.uk, which is supported by the BBSRC (grant number BB/L024209/1)) using the Nextera XT library preparation kit protocol on a HiSeq platform, generating 250 bp paired end reads (Illumina, San Diego, CA, USA). The resulting datasets are available from the SRA under BioProject number PRJEB47273. Genome sequences de novo assembled and annotated using Unicycler v 0.4.7 (28) with default parameters and Prokka v 1.14.6 (29). 
were mapped to the reference genome S. capitis AYP1020 (NCBI Genome accession GCA_001028645.1) using Snippy v.4.4.3 with minimum coverage of 4 to generate core genome SNP alignment files (https://github.com/tseemann/snippy). The phylogeny of the strains was reconstructed by generating a Maximum-likelihood (ML) tree with the substitution model GTR+G+ASC and 1000 bootstrap replicates using IQTREE v 1.6.12 (30), based on the core genome alignment without the recombining regions identified by Gubbins v2.3.4 (31). Gubbins was run with default parameters on the core genome alignment of 186 strain, which generated a a chromosomal SNP alignment length of 59,972 bp. Additionally, regions containing phage identified using PHASTER (32) and MGE sequences were identified from the reference genomes, and co-ordinates were used to mask these sites, using BEDTools v 2.29.2 (33). The population structure was investigated using hierarchical Bayesian analysis of population structure with r-hierBAPS, specifying two clusters levels, 20 initial clusters and infinite extra rounds (34). Visualisations were performed using iTOL v4.2 (35).

To measure the extent of S. capitis genomic diversity, pairwise SNP distance was determined from the alignment of core genome SNPs identified outside regions of recombination using snp-dists $v$ 0.7.0 (https://github.com/tseemann/snp-dists). To examine the phenotypic basis for the separation of the two subspecies within the phylogenetic reconstruction (Figure $2 \& 3$ ) the API Staph-Ident Stripe system was used to analyse the biochemical profiles of multiple isolates included in this study (BioMérieux, Marcy l'Etoile, France). The API Staph-Ident Strip system was used according to the manufacturer's instructions.

\section{Pangenome analysis}

Pangenome analysis of all 186 isolates was performed using the Panaroo v1.1.2 software package ran with default parameters, MAFFT alignment and a core gene threshold of $90 \%$ (36). Predicted coding gene sequences in all isolates were extracted, separated into core and accessory gene groups and input into eggnog-mapper v 2.1.6 to identify cluster of orthologs groups (COG) (37).

To identify genes enriched in S. capitis ssp. ureolytics, the output files from Panaroo were used 
Hochberg $p<0.05$ and an odds ratio $(\mathrm{OR})>1$ were considered to be overrepresented in the subspecies cluster.

\section{In silico analysis of dataset}

Genetic determinants for AMR were identified using ABRicate v0.9.8 (https://github.com/tseemann/abricate) with, NCBI Bacterial Antimicrobial Resistance Reference Gene Database (39). Other potential virulence factors, such as phenol soluble modulins and exoenzymes, as well as cell wall associated proteins and two-component systems were identified by homology searches, using BLASTp of annotated reference genomes (S. capitis AYP1020 (Genbank assembly accession: GCA_001028645.1), S. epidermidis RP62a (Genbank assembly accession: GCA_000011925.1) and S. aureus MW2 (Genbank assembly accession: GCA_00307695.1) and pangenome data from various studies $(17,40-43)$.

Average nucleotide identity (ANI) indices were used to quantify genetic relatedness of the two S. capitis subspecies. ANI estimates the genetic relatedness between two genomes to assess species boundaries. To compare the relatedness of the S. capitis subspecies in this study, FastANI v1.2 was used with default settings to compare all isolates of potential ssp. ureolyticus to ssp. capitis isolates, using the recommended cut-off score of $>95 \%$ that would indicate that isolates belong to the same species (44). S. capitis ssp. ureolyticus culture collection isolates were also compared to other ssp. ureolyticus isolates, similarly to Bannerman and Kloos (1991).

\section{Ethics approval}

Written informed consent was obtained from all participants. The study was reviewed and approved by the Independent Ethics Committee of the Unilever Port Sunlight, United Kingdom and it was conducted in compliance with the Declaration of Helsinki and its subsequent revisions.

\section{Results}

\section{Genome composition}

S. capitis sequence reads (scalp isolates, $n=59$, contextual isolates, $n=127$ ) were assembled into draft genomes with an average of 85 contigs. The average size of the assembled genomes ranged 
from 2.2-2.6 Mb. Each genome had on average 2,335 (2,087-2,565) predicted protein sequences (CDSs) with an average GC content of 32.7\%.

The pan-genome of the $186 \mathrm{~S}$. capitis isolate dataset comprised 4,471 unique clusters of orthologous groups (COGs). The pan-genome was further divided into 1,595 (35.6\%) core genes (shared by all genomes) and 2,876 (64.3\%) accessory genes (shared by $~ 95 \%$ of genomes). Gene accumulation curves reflected an open pan-genome, where the addition of each new genome increases the total gene pool (Figure 1).

The majority of annotated genes in the accessory genomes of both S. capitis ssp. capitis (53\%) and ssp. ureolyticus (57\%) were poorly characterized, this was also true of the core genome (ssp. capitis $41 \%$ and ssp. ureolyticus $38 \%$ ). This could indicate the presence of novel gene clusters in the S. capitis genome and the level of curation. The most abundant categories in both subspecies core genomes were those linked to essential gene function such as transcription (ssp. capitis $7 \%$ and ssp. ureolyticus $7 \%$ ). This is in contrast to the accessory genome where gene clusters associated with replication, recombination and repair (ssp. capitis $9 \%$ and ssp. ureolyticus $9 \%$ ) were enriched. This same pattern was separately observed in the pan-genome analysis of each subspecies (Figure 1).

\section{Population Structure and genetic diversity}

To explore the population structure of $S$. capitis, a maximum-likelihood phylogenetic tree was constructed based on chromosomal SNP alignment length of 59,972 bp. This revealed two distinct clades separated by an average pairwise distance of 7,538 core genome SNPs, with culture collection type and contextual strains of $S$. capitis ssp. capitis and S. capitis ssp. ureolyticus belonging to the upper and bottom clade, respectively (Figure 2). While the S. capitis ssp. capitis clade comprised of a single dominant subclade, $S$. capitis ssp. ureolyticus is more diverse and comprised of 3 clades. Population structure was also inferred using BAPS to cluster genomes based on shared patterns of variation, which was congruent with the phylogeny. In this study, clinical isolates are defined as those isolated from a clinical setting e.g. hospital neonatal unit or from a disease state host. Whereas commensal isolates are defined as those isolated from healthy hosts and the community. Isolate origins were overlaid onto the phylogeny and revealed clinical isolates were predominantly found in the S. capitis ssp. ureolyticus clade, while commensal isolates were associated with the S. capitis ssp. capitis clade. However, 22 
clinical isolates were observed to be interspersed across the dominant sub-clade within the $S$. capitis ssp. capitis clade and 77 commensal isolates were interspersed across two sub-clades within the $S$. capitis ssp. ureolyticus (Figure 2). This suggests that commensal and clinical isolates belonging to each subspecies are genetically similar and evolved from a common ancestor.

Extensive recombination was observed among the study isolates, with recombination most evident in BAPS clusters 2, 3, 4 and 5, which collectively contain 293 recombination blocks. BAPS cluster 1 revealed less recombination (total of 2 recombination blocks), however this is likely due to the reference genome itself being clustered in this group. Recombination was inferred across large regions of the chromosome, predominantly within the first $\sim 750 \mathrm{~kb}$ and the last $\sim 1 \mathrm{Mb}$ of the genome (Figure S1). The recombination data is consistent with $S$. capitis having arisen following extensive recombination events (13).

\section{Insights into $S$. capitis pathogenicity}

The CoNS that colonise human skin are generally considered to be non-pathogenic species specialised for healthy human skin and mucosal surfaces, but they are now emerging as important opportunistic pathogens $(20,22,24,45-47)$. Antimicrobial resistance properties are important factors of nosocomial infection. Therefore, we screened for presence of genetic determinants for AMR and identified genes predicted to encode for resistance against tetracyline, $\beta$-lactam, bleomycin, fosfomycin, methicillin resistance, fusidic acid streptogramin A, macrolide, linezolid, trimethoprim and aminoglycoside. Amongst the S. capitis genomes analysed, $48 \%$ (5\% S. capitis ssp. capitis and $44 \%$ S. capitis ssp. ureolyticus) were classified as MDR, carrying genetic determinants conferring resistance against three or more classes of drugs (Figure 2). MDR was found in $78 \%$ of isolates from the ureolyticus clade, compared to $11 \%$ of isolates from the capitis clade. When the dataset was stratified by isolate origin, we found clinical isolates carried more AMR genes than compared to commensal. A total of 635 AMR linked genes were found across clinical isolates $(n=99)$ compared to 169 found in commensal isolates $(n=81)$.

In addition to AMR genes, we also investigated the role of phenol-souble modulins (PSMs) contributing to the virulence potential of $S$. capitis. PSMs are a novel toxin family that have 
antimicrobial activity $(48,49)$ and have been attributed to the competitive success of CoNS due to their ability to inhibit the growth of other commensal bacteria such as Cutibacterium acnes (O'Neill et al., 2020). A total of 5 gene clusters encoding $\beta$-class PSMs were identified (Figure 3), with gene clusters 1634, 1469 and 2040 found in $>98 \%$ of isolates, sharing $>90 \%$ similarity when locally aligned to PSMs described and isolated from S. capitis, by O'Neill et al., (O’Neill et al., 2020). Similar to AMR gene presence and absence, PSM-associated gene clusters were found more abundantly in the S. capitis ssp. ureolyticus clade than compared with the S. capitis ssp. capitis clade. Specifically, gene clusters 1421 and 1474 were found in $66 \%$ and $55 \%$ of isolates from the ureolyticus clade, in contrast to $3 \%$ and $0 \%$ isolates from the capitis clade. (Figure 4).

To further investigate variation of the $\beta$-class PSMs identified, we performed multiple sequence alignment of corresponding amino acid sequence from the 6 gene clusters, which revealed conservation of residues attributed to the maintenance of the amphipathic nature of the peptides, essential to PSM antimicrobial activity (50). Specifically, lysine at $3^{\text {rd }}$ and/or tryptophan at $20^{\text {th }}$ position are putatively associated with providing antibacterial activity of $\beta$-class family peptides (50), both of which are conserved in the peptide sequences of this study (Figure 4).

Additionally, we specifically screened for the presence of orthologous CDS that likely contribute to S. capitis pathogenicity. Including, staphylococcal cell wall associated (CWA) proteins curated with potential virulence roles, including biofilm-associated proteins IcaRADBC, capsule biosynthesis proteins CapDACB, surface adhesins AtlE, Pls, Aap, FnbpA, SesA, SesB, SesC, SesG, Ebp and Bap, and MSCRAMMs SdrX, SdrZL, SdrH, SdrF and SdrG. Of these CWA proteins, 11 were encoded in S. capitis AYP1020. Across the 186 S. capitis genomes analysed, sesA, sesB, sesC, sesG, icaRADBC, fbnpA, capDACB and atlE were found in all isolates. MSCRAMM genes $s d r X$ and $s d r Z L$ were found in $>95 \%$ of genomes investigated. Contrastingly, the surface adhesin gene $p l s$ was found in $<10 \%$ of $S$. capitis genomes and was absent from ssp. capitis (Figure 3). Notably, genes that were absent from the S. capitis AYP1020 genome (determined in this study as S. capitis ssp. ureolyticus) but present in other CoNS species such as, S. epidermidis RP62a (Cameron et al., 2015), included $s d r F$, 
study (17) (Figure 3). The secreted protein genes hlb, clpP, clpBCX, sepA, htrA, lip, gehl, geh2 and lipA were present in $>95 \%$ of all the isolates. The presence of a suite of exoproteins could contribute to host colonisation, persistence, infection and immune evasion, important to both pathogenesis and colonisation (22). Notably, $s p A$, $\operatorname{sp} B$ and $\operatorname{ssp} C$ were absent in $S$. capitis genomes, the serine protease SspA promotes invasion in S. aureus (51) (Figure 3).

The 16 two component systems (TCS) described in S. aureus are conserved in other closely related CoNS species, indicative of an adaptive and highly versatile pathogen (52, 53). S. aureus TCS are extensively characterised and therefore the $186 \mathrm{~S}$. capitis genomes included in this study were screened for homologous protein sequences. Of the $16 \mathrm{TCS}, 14$ were found in S. capitis. This is intermediate with S. epidermidis (16 TCS) and S. saprophyticus (11 TCS) with number determined by Rapun-Araiz, et al. (2020) to be indeterminate of genome size in staphylococci (Table 1).

\section{S. capitis subspecies definition}

To biochemically assess differences between the two subspecies $S$. capitis ssp. capitis and $S$. capitis ssp. ureolyticus based upon the original descriptions of Bannerman and Kloos (2) the API StaphIdent Strip system was used (BioMérieux, Marcy l'Etoile, France). A total of 22 isolates sampled in this study that were spread across the phylogenetic tree (11 assigned to the S. capitis ssp. capitis clade and 11 assigned to the $S$. capitis ssp. ureolyticus clade), as well as type and culture collection stains were tested for classifying phenotypic traits. Among the 11 isolates belonging to the $S$. capitis ssp. ureolyticus clade, only 4 (33\%) tested urease-positive/maltose-positive and 10 (91\%) S. capitis ssp. capitis isolate tested urease-negative/maltose-negative, indicating an unreliable phenotype of urease activity and maltose fermentation as a subspecies definition (data not shown).

Since the original phenotypic trait descriptors for S. capitis did not sufficiently discriminate between the subspecies, we then sought to quantify and analyse the genetic differences between $S$. capitis ssp. ureolyticus and capitis. Analysis of the average nucleotide identity (ANI) between isolates determined here as $S$. capitis ssp. ureolyticus and S. capitis ssp. capitis revealed that genomes from the two subspecies shared little genetic differences with 96\% ANI. Pan-genomic comparative analysis also revealed limited gene content variation of $1 \%$ between the two subspecies. To help identify 
genes that could be used to discriminate between the subspecies and serve as diagnostic markers

270 for rapid identification by PCR in future studies, we looked for genes which are significantly overrespresented in each subspecies and identified total of 38 gene clusters found across all $S$. capitis ssp. capitis genomes and 13 across all S. capitis ssp. ureolyticus genomes (Table 2, Supplementary Table $2 \& 3$ ). Upon closer inspection, majority of the subspecies unique genes identified were in fact divergent gene orthologues. An example of this is the icaC gene, which encodes for an intercellular adhesion protein. While one version of this gene cluster was only found in S. capitis ssp. ureolyticus isolates (98\%), another version sharing blastp identity of $22 \%$ was only present in S. capitis ssp. capitis isolates $(0 \%)$.

To further investigate specific genetic signatures associated with each $S$. capitis subspecies, we applied Scoary to identify genes that are overrepresented in S. capitis ssp. capitis, as well as S. capitis ssp. ureolyticus. A total of 1,086 predicted gene clusters were found to differ significantly $(p<0.05)$ between the subspecies, although there were no significant differences found between assigned functional COG categories (Table 1, Supplementary Table 2, Figure 1). Gene clusters with a known function identified as being enriched in S. capitis ssp. capitis isolates include those for the arginine catabolic mobile element (ACME), encoding arginine deiminase activity found in various species of staphylococci. Most S. capitis isolates in this study contain a Type V ACME gene cluster, however different conserved versions are found in each subspecies. Additional gene clusters enriched in ssp. capitis included those encoding: SasC cell wall anchored protein; and $\mathrm{CrtN}$ dehydrosqualene desaturase involved in staphyloxanthin biosynthesis (Table 2, Supplementary Table $2 \&$ 3). For S. capitis ssp. ureolyticus, genes predicted to encode for virulence factor were found to be enriched. Specifically, genes with antimicrobial-associated functions including $\beta$-lactam resistance protein BlaR and $\beta$-class phenol soluble modulins (Table 2, Supplementary Table $2 \& 3$ ). This is concurrent with the current 


\section{Discussion}

S. capitis is an opportunistic pathogen that is associated with increasing reports of bloodstream infections and neonatal infections in intensive care units. Currently, S. capitis is mostly studied with reference to the well-described and clinically important $S$. epidermidis. In the absence of an expansive study of S. capitis genomes from the scalp, the current work aimed to explore WGS of the species to expand knowledge of its population structure, compare and contrast genomic differences between commensal and clinical isolates to gain understanding of the genetic factors that contributes to S. capitis pathogenicity.

Pangenome analysis indicated that the $S$. capitis has an open pangenome, which could arise from a capacity to acquire exogenous DNA whilst living in extensive bacterial communities. The large accessory genome size suggests that $S$. capitis contains a large repertoire of genes that confer advantages under particular environmental conditions to support its colonisation and/or cause infections in clinical settings. Similarly, other members of the Epidermidis cluster group, such as $S$. caprae and $S$. epidermidis also have a large, open pangenome state compared to other CoNS- Staphyloccus lugeunensis $(54,55)$. It can therefore be hypothesised that $S$. capitis, like other staphylococci, undergo horizontal gene transfer (HGT) which could also have led to the acquisition of virulence genes within S. capitis genomes (54). The identification of more virulent strains of S. capitis ssp. ureolyticus, in 311 greater frequencies in clinical settings combined with less virulent strains isolated from other sources, such as the scalp could indicate a potential contextual basis for the HGT events, although further investigation is needed to understand the association of S. capitis subspecies and scalp skin state. Many studies using the higher resolution afforded by WGS have enabled important differences to be uncovered in S. capitis genomes, such as their multidrug resistance profiles across different geographic regions $(5,13,17)$. subspecies, herein termed the $S$. capitis spp. capitis and the S. capitis spp. ureolyticus clade. Most of the clinical isolates from this study belonged to the S. capitis spp. ureolyticus clade and commensal isolates belonging to the $S$. capitis spp. capitis clade, suggesting that $S$. capitis spp. ureolyticus is more 
associated with clinical infections. This agrees with the current literature that suggests $S$. capitis spp. ureolyticus is the more virulent subspecies and linked to the presence of genes linked to biofilm formation and methicillin resistance $(5,8)$. However, the observation of clinical isolates interspaced across the dominant sub-clade of $S$. capitis spp. capitis clade indicates that clinical and commensal isolates share similar genetic background, and while S. capitis spp. capitis is more associated with commensal it can cause clinical infection.

Although the scalp-associated isolates sampled in this study were not from clinical infections, potential virulence-linked genes were found throughout their genomes, highlighting $S$. capitis versatility and potential for adaptation that might cause significant disease in settings like the NICU. Further exploration of sequence differences will be required to unravel defining features of $S$. capitis subspecies to support the hypothesis that $S$. capitis ssp. ureolyticus or genomes belonging to the $S$. capitis ssp. ureolyticus clade, are generally more virulent.

Most published studies have focused on S. capitis strains isolated from NICU and other clinical settings, describing the emergence of drug resistance in response to the use of antimicrobial and antiseptic therapy to treat infections caused by CoNS isolates $(13-15,56,57)$. Antimicrobial resistance to vancomycin and fusidic acid has been reported among S. capitis, like $S$. aureus, suggesting the occurrence of inter-species genetic exchange $(13,56)$.

In addition to antimicrobial resistance, biofilm formation was proposed to be an important virulence trait of $S$. capitis in both a clinical and commensal setting $(5,20)$. In keeping with this proposal, the current work confirms the presence of biofilm-related genes in the $S$. capitis genomes studied, including icaRADBC operon, $e b h$ and $a t l E$, and extends it by identifying an IcaC encoding gene cluster discriminating the subspecies with its presence in ssp. ureolyticus. The role of IcaC (Table 2) in S. capitis has been described by Cui, et al as modifying synthesised glucan by acetylation (2015). A more extensive investigation, into IcaC, including S. capitis isolated from different sources could contribute to further understanding trait differences that determine ssp. specialisation. The suite of biofilm genes facilitate staphylococcal primary attachment by binding to extracellular matrix molecules, and intercellular aggregation (20). The presence of these genes may confer a selective advantage in both a clinical setting and on the scalp and forehead. Further phenotypic studies of biofilm 
formation, metabolism, and multidrug resistance in S. capitis isolates, including those in this study will extend our knowledge of speciation and specialisation of staphylococci and S. capitis, and could help with precise studies of factors that pertain to emergent clinical disease. descriptions of $S$. capitis ssp. ureolyticus urease activity, ability to produce acid from maltose, fatty acid profile, larger colony size and DNA sequence differentiation (2). The multiple discriminating traits were not explored in full here but biochemical analysis using API Staph-Ident Strip system, the most common method to discriminate $S$. capitis subspecies, was tested on 14 isolates sampled from this study and 3 isolates from the culture collection type strains. Discrepancy between biochemical and whole genome phylogenetic assignment of the subspecies were observed, as only $33 \%$ of tested isolates that belonged to the S. capitis ssp. ureolyticus clade tested positive for urease activity. Highlighting that classification of $S$. capitis subspecies by urease activity is unreliable and requires confirmation using other discriminating traits.

Further analysis to characterise the genetic relatedness between the two subspecies using ANI revealed genomes of each subspecies were similar, sharing $96 \%$ nucleotide identity. Instead, we investigated discriminating gene clusters and observed that $S$. capitis spp. ureolyticus genomes were enriched with antimicrobial resistance gene functions, such as $\beta$-lactam resistance genes and $\beta$ - class phenol soluble modulins. Whereas S. capitis spp. capitis genomes were enriched with gene clusters linked to skin survival based on the presence of the arginine catabolism mobile element (ACME) that encodes enzymes to counteract low $\mathrm{pH}(58,59)$. ACME is a genomic island first described in S. aureus USA300 and in S. epidermidis ATCC $12228(60,61)$. It was shown to enhance staphylococcal colonisation of the skin and mucous membranes, showing similar characteristics to the staphylococcal cassette chromosome mec (SCCmec) element $(62,63)$. While not investigated functionally here in $S$. capitis it is likely to have a similar function. We hypothesise that ACME activity, that discriminates ssp. capitis, could represent a key factor of subspeciation through niche specialisation. Analysis of virulence gene profiles determined that as a species, S. capitis has a similar repertoire of virulence genes to several other CoNS species, with respect to AMR, PSMs and secreted proteases $(54,64)$. 
required for robust markers of subspecies classification within their core genomes though several genes exclusive to every isolate of each subspecies were identified and could serve as subspecies biomarkers.

In conclusion, this study identified distinct clustering of the two subspecies of S. capitis and determined gene clusters for traits that might rapidly progress our understanding of $S$. capitis relevant to disease. Specifically, we propose that the original subspecies definition ssp. ureolyticus needs reconsidered based on species subclades that define it based on the importance of MDR and virulence. It is likely that the widespread use of antimicrobials, the openness of the S. capitis pangenome and acquisition of MGEs with beneficial mutations has promoted the emergence of virulence traits in $S$. capitis isolates. Continued research into classification of S. capitis as subspecies versus virulence linked phylogroups is important for better surveillance of the spread and evolution of S. capitis.

\section{Data Summary}

Short read sequences supporting the findings of this study have been deposited in the European Nucleotide Archive (https://www.ebi.ac.uk/ena/) under the project accession number PRJEB47273. Accession numbers for isolates used in this study are listed in Supplementary Table 1. Publicly available sequences were downloaded from GenBank (https://www.ncbi.nlm.nih.gov/genbank/), Sequence Read Archive (https://www.ncbi.nlm.nih.gov/sra) or European Nucleotide Archive (https://www.ebi.ac.uk/ena), and accession numbers are listed in Table Supplementary Table 1.

\section{Funding}

This work was funded by a Biotechnology and Biological Sciences Research Council Doctoral Training Partnership CASE studentship BB/M011186/1 awarded to Dr M. J. Horsburgh, with support from Unilever PLC. Unilever R\&D, Port Sunlight, were involved in the study design for collection of scalp samples included in this study.

\section{Conflict of Interest Statement}

The authors declare that the research was conducted in the absence of any commercial or financial relationships that could be construed as a potential conflict of interest. 
402

\section{Acknowledgments}

DNA sequencing was performed by the Centre for Genomic Research, Liverpool, UK. We are grateful to Sally Grimshaw for technical assistance and Barry Murphy for technical discussions.

\section{Author Notes}

C.E.C performed all the data analysis and interpretation for the results under the scientific guidance of R.J.B and M.J.H. C.E.C and M.J.H drafted the manuscript. All authors contributed to editing of the manuscript.

All supporting data, code and protocols have been provided within the article or through supplementary data files. Three supplementary tables and one supplementary figure are available.

\section{Figure Legends}

Figure 1. Representation of the pangenome and COG functional annotation of $S$. capitis genomes.

(A) Pangenome curve generated by plotting total number of gene families in the pan (blue) and core (red) genome of S. capitis. The graph represents how the pan- and core- genomes vary as genomes are added in random order. As the number of genomes increased, the pan-genome increased. (B) Staphylococcus capitis pangenome statistics. The size of the pangenome, including core (shared by $>95$ $\%$ of isolates), shell (found in $15-95 \%$ of isolates) and cloud (found in $<15 \%$ of isolates) genes. (C) Functional annotation of the core and accessory genomes of S. capitis subsp. capitis and S. capitis subsp. ureolyticus. Percentages of the core and accessory genomes annotated according to COG functional categories.

Figure 2. Maximum-likelihood phylogeny based on core genome alignments of 186 . capitis isolates, presenting the presence and absence of antimicrobial resistance genes. ML tree is midpoint rooted and bootstrap support values were calculated from 1,000 replicates. The first colour block represents rhierBAPS clustering, dots describe the setting where isolates were retrieved; green = commensal (including scalp samples from this study), red = clinical and grey = unknown and filled grey triangles describe scalp isolates from this study. The subspecies differentiation of S. capitis is presented as the subclades described as BAPS groups 1, 3, 4 and 5. Presence (coloured blocks) and absence (white 
blocks) of antimicrobial resistance is denoted for each isolate (*Quaternary Ammonium Compounds).

The scale bar represents the number of nucleotide substitutions per site. Figure was visualised using

iTol v $4.2(35)$.

Figure 3. Maximum-likelihood phylogeny based on core genome alignments of 186 S. capitis block represents rhierBAPS clustering, dots describe the setting where isolates were retrieved; green = commensal (including scalp samples from this study), red = clinical and grey = unknown and filled grey triangles describe scalp isolates from this study. The subspecies differentiation of $S$. capitis is presented as the subclades described as BAPS groups 1, 3, 4 and 5. Presence (coloured blocks) and absence (white blocks) of virulence genes is denoted for each isolate. The scale bar represents the number of nucleotide substitutions per site. Figure was visualised using iTol v 4.2 (35).

Figure 4. Multiple sequence alignment of $\beta$-class Phenol Soluble Modulins (PSMs) of $S$. capitis isolates. MSA of $\beta$-class PSMs protein sequences found in S. capitis genomes from this study and those described by O'Neill et al., (2020) (sequences marked with $\mathbf{\Delta}$ ) created with ClustalW (65). Residues are coloured based on amino acid property (Red: small and hydrophobic, blue: acidic, magenta: basic, green: hydroxyl, sulfhydryl, and amine and grey: unusual), positions that contain fully conserved residues are marked with and asterisk and positions marked with a colon indicate conservation between groups of amino acids with similar properties.

\section{Table legends}

Table 1. Two-component systems in S. aureus and S. capitis. Presence and absence of the 16 TCS of S. aureus (MW2) described in S. capitis reference genome AYP1020 and isolates included in this study.

Table 2. Gene clusters found significantly enriched in either S. capitis ssp. capitis or ssp. ureolyticus ( $\boldsymbol{p}<\mathbf{0 . 0 0 1})$. Gene clusters, presence and absence, and functional descriptions were obtained from Panaroo and Scoary pangenome analysis of assembled genomes. $\mathrm{X}$ these genes exist in different 
(Genbank Assembly Accession: GCA_001028645.1) (17). The complete list can be found at

\section{Supplementary Information}

Figure S1. Analysis of the $S$. capitis genome alignment with Gubbins. The maximum likelihood

phylogenetic reconstruction of $S$. capitis is shown on the left, with coloured bands highlighting rhierBAPS clustering and isolation site of genomes. Filled grey triangles describe scalp isolates from this study. Homologous recombination events for each S. capitis genome ordered based on their position in the AYP1020 reference genome (shown along the top) are shown on the right. Recombination blocks detected in $>1$ isolate are shown in red, while blocks affecting a single isolate are indicated in blue. Figure visualised using Phandango (66).

Table S1. Staphylococcus capitis isolates used in this study. Isolates were either isolated in this study, type strains or sequence data obtained from the NCBI database.

Table S2. Genes significantly differentiating $S$. capitis subspecies. Gene identification output from Scoary analysis.

Table S3. Representative protein sequences of hypothetical gene clusters that significantly differentiate $S$. capitis subspecies. Gene identification output from Scoary analysis and protein sequence information from annotated genome assemblies.

\section{References}

1. Kloos WE, Schleifer KH. Isolation and characterization of staphylococci from human skin II. descriptions of four new species: Staphylococcus warneri, Staphylococcus capitis, Staphylococcus hominis, and Staphylococcus simulans1. International Journal of Systematic and Evolutionary Microbiology. 1975;25(1):62-79.

477 2. Bannerman TL, Kloos WE. Staphylococcus capitis subsp. ureolyticus subsp. nov. from human skin. Int J Syst Bacteriol. 1991;41(1):144-7. human subjects in isolation and clonal analysis of Staphylococcus capitis by sodium dodecyl sulfate-polyacrylamide gel electrophoresis. J Clin Microbiol. 1989;27(12):2627-32. abundance of fungi and bacteria on the healthy and dandruff affected human scalp. PLoS One. 2019;14(12):e0225796. 

clinical Staphylococcus capitis subspecies as revealed by biofilm, antibiotic resistance, and pulsed-field gel electrophoresis profiling. Journal of clinical microbiology. 2013;51(1):9-14. 6. Cui B, Smooker PM, Rouch DA, Deighton MA. Effects of erythromycin on the phenotypic and genotypic biofilm expression in two clinical Staphylococcus capitis subspecies and a functional analysis of Ica proteins in S. capitis. Journal of medical microbiology. 2015;64(6):591-604.

7. Tevell S, Hellmark B, Nilsdotter-Augustinsson Å, Söderquist B. Staphylococcus capitis isolated from prosthetic joint infections. Eur J Clin Microbiol Infect Dis. 2017;36(1):115-22.

4948 8. Tevell S, Baig S, Hellmark B, Martins Simoes P, Wirth T, Butin M, et al. Presence of the neonatal Staphylococcus capitis outbreak clone (NRCS-A) in prosthetic joint infections. Scientific Reports. 2020;10(1):22389. 9. Flurin L, Greenwood-Quaintance KE, Patel R. Microbiology of polymicrobial prosthetic joint infection. Diagnostic Microbiology and Infectious Disease. 2019;94(3):255-9. 10. Nalmas S, Bishburg E, Meurillio J, Khoobiar S, Cohen M. Staphylococcus capitis prosthetic valve endocarditis: Report of two rare cases and review of literature. Heart \& Lung. 2008;37(5):380-4.

502 11. Cone LA, Sontz EM, Wilson JW, Mitruka SN. Staphylococcus capitis endocarditis due to a transvenous endocardial pacemaker infection: Case report and review of Staphylococcus capitis endocarditis. International Journal of Infectious Diseases. 2005;9(6):335-9.

505 12. Al Hennawi HET, Mahdi EM, Memish ZA. Native valve Staphylococcus capitis infective endocarditis: a mini review. Infection. 2020;48(1):3-5. 13. Carter GP, Ussher JE, Da Silva AG, Baines SL, Heffernan H, Riley TV, et al. Genomic Analysis of Multiresistant Staphylococcus capitis Associated with Neonatal Sepsis. Antimicrobial agents and chemotherapy. 2018;62(11):e00898-18. specialization and spread of Staphylococcus capitis involved in neonatal sepsis. Nature Microbiology. 2020;5(5):735-45.

513 15. Rasigade J-P, Raulin O, Picaud J-C, Tellini C, Bes M, Grando J, et al. Methicillin514 Resistant Staphylococcus capitis with Reduced Vancomycin Susceptibility Causes Late-Onset Sepsis in Intensive Care Neonates. PLoS One. 2012;7(2):e31548. 16. Stenmark B, Hellmark B, Söderquist B. Genomic analysis of Staphylococcus capitis isolated from blood cultures in neonates at a neonatal intensive care unit in Sweden. European Journal of Clinical Microbiology \& Infectious Diseases. 2019;38(11):2069-75.

519 17. Cameron D, Jiang J-H, Hassan K, Elbourne L, Tuck K, Paulsen I, et al. Insights on virulence from the complete genome of Staphylococcus capitis. Frontiers in Microbiology. 2015;6(980).

18. Lamers RP, Muthukrishnan G, Castoe TA, Tafur S, Cole AM, Parkinson CL. Phylogenetic relationships among Staphylococcus species and refinement of cluster groups based on multilocus data. BMC Evol Biol. 2012;12:171. genome sequencing of three human clinical isolates of Staphylococcus caprae reveals virulence factors similar to those of S. epidermidis and S. capitis. BMC Genomics. 2018;19(1):810. 20. Otto M. Staphylococcus epidermidis — the 'accidental' pathogen. Nature Reviews Microbiology. 2009;7(8):555-67.

21. Massey RC, Horsburgh MJ, Lina G, Höök M, Recker M. The evolution and maintenance of virulence in Staphylococcus aureus: a role for host-to-host transmission? Nature Reviews Microbiology. 2006;4(12):953-8.

22. Heilmann C, Ziebuhr W, Becker K. Are coagulase-negative staphylococci virulent? Clinical Microbiology and Infection. 2019;25(9):1071-80. 
23. Becker K, Heilmann C, Peters G. Coagulase-Negative Staphylococci. Clinical Microbiology Reviews. 2014;27(4):870.

24. Otto M. Coagulase-negative staphylococci as reservoirs of genes facilitating MRSA infection: Staphylococcal commensal species such as Staphylococcus epidermidis are being recognized as important sources of genes promoting MRSA colonization and virulence. Bioessays. 2013;35(1):4-11.

25. Argemi X, Hansmann Y, Prola K, Prévost G. Coagulase-Negative Staphylococci Pathogenomics. International journal of molecular sciences. 2019;20(5):1215.

26. Williamson P, Kligman AM. A new method for the quantitative investigation of cutaneous bacteria. The Journal of investigative dermatology. 1965;45(6):498-503.

27. Menardo F, Loiseau C, Brites D, Coscolla M, Gygli SM, Rutaihwa LK, et al. Treemmer: a tool to reduce large phylogenetic datasets with minimal loss of diversity. BMC Bioinformatics. 2018;19(1):164.

28. Wick RR, Judd LM, Gorrie CL, Holt KE. Unicycler: Resolving bacterial genome assemblies from short and long sequencing reads. PLoS computational biology. 2017;13(6):e1005595-e.

29. Seemann T. Prokka: rapid prokaryotic genome annotation. Bioinformatics. 2014;30(14):2068-9.

30. Chernomor O, von Haeseler A, Minh BQ. Terrace Aware Data Structure for Phylogenomic Inference from Supermatrices. Systematic Biology. 2016;65(6):997-1008.

31. Croucher NJ, Page AJ, Connor TR, Delaney AJ, Keane JA, Bentley SD, et al. Rapid phylogenetic analysis of large samples of recombinant bacterial whole genome sequences using Gubbins. Nucleic Acids Research. 2014;43(3):e15-e.

32. Arndt D, Grant JR, Marcu A, Sajed T, Pon A, Liang Y, et al. PHASTER: a better, faster version of the PHAST phage search tool. Nucleic Acids Res. 2016;44(W1):W16-21.

33. Quinlan AR, Hall IM. BEDTools: a flexible suite of utilities for comparing genomic features. Bioinformatics. 2010;26(6):841-2.

34. Cheng L, Connor TR, Sirén J, Aanensen DM, Corander J. Hierarchical and Spatially Explicit Clustering of DNA Sequences with BAPS Software. Molecular Biology and Evolution. 2013;30(5):1224-8.

35. Letunic I, Bork P. Interactive tree of life (iTOL) v3: an online tool for the display and annotation of phylogenetic and other trees. Nucleic Acids Res. 2016;44(W1):W242-5.

36. Tonkin-Hill G, MacAlasdair N, Ruis C, Weimann A, Horesh G, Lees JA, et al. Producing polished prokaryotic pangenomes with the Panaroo pipeline. Genome Biology. 2020;21(1):180.

37. Huerta-Cepas J, Forslund K, Coelho LP, Szklarczyk D, Jensen LJ, von Mering C, et al. Fast Genome-Wide Functional Annotation through Orthology Assignment by eggNOGMapper. Mol Biol Evol. 2017;34(8):2115-22.

38. Brynildsrud O, Bohlin J, Scheffer L, Eldholm V. Rapid scoring of genes in microbial pan-genome-wide association studies with Scoary. Genome biology. 2016;17(1):1-9.

39. Feldgarden M, Brover V, Haft DH, Prasad AB, Slotta DJ, Tolstoy I, et al. Validating the AMRFinder Tool and Resistance Gene Database by Using Antimicrobial Resistance Genotype-Phenotype Correlations in a Collection of Isolates. Antimicrobial agents and chemotherapy. 2019;63(11):e00483-19.

40. Gill Steven R, Fouts Derrick E, Archer Gordon L, Mongodin Emmanuel F, DeBoy Robert T, Ravel J, et al. Insights on Evolution of Virulence and Resistance from the Complete Genome Analysis of an Early Methicillin-Resistant Staphylococcus aureus Strain and a Biofilm-Producing Methicillin-Resistant Staphylococcus epidermidis Strain. Journal of Bacteriology. 2005;187(7):2426-38. 
41. Baba T, Bae T, Schneewind O, Takeuchi F, Hiramatsu K. Genome sequence of Staphylococcus aureus strain newman and comparative analysis of staphylococcal genomes: Polymorphism and evolution of two major pathogenicity islands. Journal of Bacteriology. 2008;190(1):300-10.

42. Baba T, Takeuchi F, Kuroda M, Yuzawa H, Aoki K-i, Oguchi A, et al. Genome and virulence determinants of high virulence community-acquired MRSA. The Lancet. 2002;359(9320):1819-27.

43. Altschul SF, Gish W, Miller W, Myers EW, Lipman DJ. Basic local alignment search tool. J Mol Biol. 1990;215(3):403-10.

44. Jain C, Rodriguez-R LM, Phillippy AM, Konstantinidis KT, Aluru S. High throughput ANI analysis of $90 \mathrm{~K}$ prokaryotic genomes reveals clear species boundaries. Nature Communications. 2018;9(1):5114.

45. Post V, Harris LG, Morgenstern M, Mageiros L, Hitchings MD, Méric G, et al. Comparative Genomics Study of Staphylococcus epidermidis Isolates from OrthopedicDevice-Related Infections Correlated with Patient Outcome. Journal of clinical microbiology. 2017;55(10):3089-103.

46. Méric G, Mageiros L, Pensar J, Laabei M, Yahara K, Pascoe B, et al. Diseaseassociated genotypes of the commensal skin bacterium Staphylococcus epidermidis. Nature Communications. 2018;9(1):5034.

47. Espadinha D, Sobral RG, Mendes CI, Méric G, Sheppard SK, Carriço JA, et al. Distinct Phenotypic and Genomic Signatures Underlie Contrasting Pathogenic Potential of Staphylococcus epidermidis Clonal Lineages. Frontiers in Microbiology. 2019;10(1971).

48. Cheung GYC, Joo H-S, Chatterjee SS, Otto M. Phenol-soluble modulins - critical determinants of staphylococcal virulence. FEMS Microbiology Reviews. 2014;38(4):698-719. 49. Peschel A, Otto M. Phenol-soluble modulins and staphylococcal infection. Nature Reviews Microbiology. 2013;11(10):667-73.

50. Kumar R, Jangir PK, Das J, Taneja B, Sharma R. Genome Analysis of Staphylococcus capitis TE8 Reveals Repertoire of Antimicrobial Peptides and Adaptation Strategies for Growth on Human Skin. Scientific reports. 2017;7(1):10447-.

51. Dubin G, Chmiel D, Mak P, Rakwalska M, Rzychon M, Dubin A. Molecular cloning and biochemical characterisation of proteases from Staphylococcus epidermidis. Biological chemistry. 2001;382(11):1575-82.

52. Haag AF, Bagnoli F. The Role of Two-Component Signal Transduction Systems in Staphylococcus aureus Virulence Regulation. In: Bagnoli F, Rappuoli R, Grandi G, editors. Staphylococcus aureus: Microbiology, Pathology, Immunology, Therapy and Prophylaxis. Cham: Springer International Publishing; 2017. p. 145-98.

53. Rapun-Araiz B, Haag AF, Solano C, Lasa I. The impact of two-component sensorial network in staphylococcal speciation. Current Opinion in Microbiology. 2020;55:40-7.

54. Sun Z, Zhou D, Zhang X, Li Q, Lin H, Lu W, et al. Determining the Genetic Characteristics of Resistance and Virulence of the "Epidermidis Cluster Group" Through PanGenome Analysis. Front Cell Infect Microbiol. 2020;10:274-.

55. Argemi X, Matelska D, Ginalski K, Riegel P, Hansmann Y, Bloom J, et al. Comparative genomic analysis of Staphylococcus lugdunensis shows a closed pan-genome and multiple barriers to horizontal gene transfer. BMC genomics. 2018;19(1):621-.

56. Chong J, Caya C, Lévesque S, Quach C. Heteroresistant Vancomycin Intermediate Coagulase Negative Staphylococcus in the NICU: A Systematic Review. PLoS One. 2016;11(10):e0164136.

57. Mello D, Daley AJ, Rahman MS, Qu Y, Garland S, Pearce C, et al. Vancomycin Heteroresistance in Bloodstream Isolates of $<$ em $>$ Staphylococcus capitis $</$ em\&gt. Journal of Clinical Microbiology. 2008;46(9):3124. 
634 58. Lindgren JK, Thomas VC, Olson ME, Chaudhari SS, Nuxoll AS, Schaeffer CR, et al. 635 Arginine Deiminase in Staphylococcus epidermidis Functions To Augment Biofilm 636 Maturation through pH Homeostasis. Journal of Bacteriology. 2014;196(12):2277-89.

637 59. Planet PJ, LaRussa SJ, Dana A, Smith H, Xu A, Ryan C, et al. Emergence of the 638 Epidemic Methicillin-Resistant Staphylococcus aureus Strain USA300 Coincides with 639 Horizontal Transfer of the Arginine Catabolic Mobile Element and $\langle\mathrm{i}\rangle$ speG $\langle/ \mathrm{i}\rangle$-mediated 640 Adaptations for Survival on Skin. mBio. 2013;4(6):e00889-13.

641 60. Planet PJ, Diaz L, Kolokotronis S-O, Narechania A, Reyes J, Xing G, et al. Parallel 642 Epidemics of Community-Associated Methicillin-Resistant Staphylococcus aureus USA300 643 Infection in North and South America. The Journal of Infectious Diseases. 2015;212(12):187464482.

645 61. Diep BA, Gill SR, Chang RF, Phan TH, Chen JH, Davidson MG, et al. Complete 646 genome sequence of USA300, an epidemic clone of community-acquired meticillin-resistant 647 Staphylococcus aureus. The Lancet. 2006;367(9512):731-9.

648 62. Diep BA, Stone GG, Basuino L, Graber CJ, Miller A, des Etages S-A, et al. The 649 Arginine Catabolic Mobile Element and Staphylococcal Chromosomal Cassette mec Linkage: 650 Convergence of Virulence and Resistance in the USA300 Clone of Methicillin-Resistant 651 Staphylococcus aureus. The Journal of Infectious Diseases. 2008;197(11):1523-30.

652 63. Almebairik N, Zamudio R, Ironside C, Joshi C, Ralph JD, Roberts AP, et al. Genomic 653 Stability of Composite SCCmec ACME and COMER-Like Genetic Elements in 654 Staphylococcus epidermidis Correlates With Rate of Excision. Frontiers in Microbiology. $6552020 ; 11(166)$.

656 64. Moawad AA, Hotzel H, Awad O, Roesler U, Hafez HM, Tomaso H, et al. Evolution of 657 Antibiotic Resistance of Coagulase-Negative Staphylococci Isolated from Healthy Turkeys in 658 Egypt: First Report of Linezolid Resistance. Microorganisms. 2019;7(10):476.

659 65. Thompson JD, Higgins DG, Gibson TJ. CLUSTAL W: improving the sensitivity of 660 progressive multiple sequence alignment through sequence weighting, position-specific gap 661 penalties and weight matrix choice. Nucleic Acids Res. 1994;22(22):4673-80.

662 66. Hadfield J, Croucher NJ, Goater RJ, Abudahab K, Aanensen DM, Harris SR. 663 Phandango: an interactive viewer for bacterial population genomics. Bioinformatics. 664 2017;34(2):292-3. 


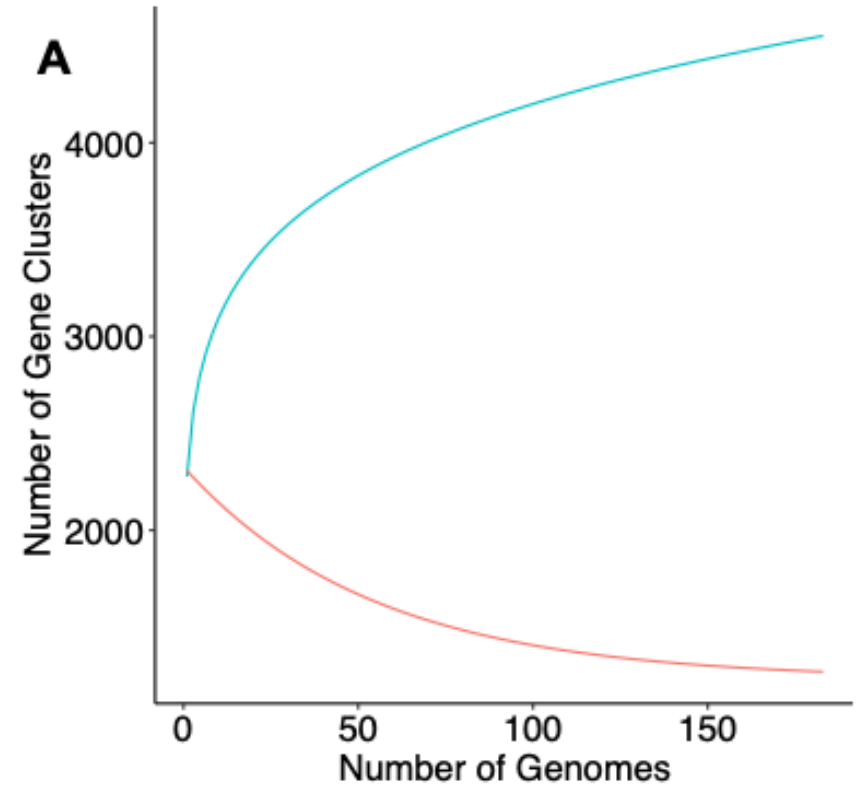

- Core-Genome Size - Pan-Genome Size

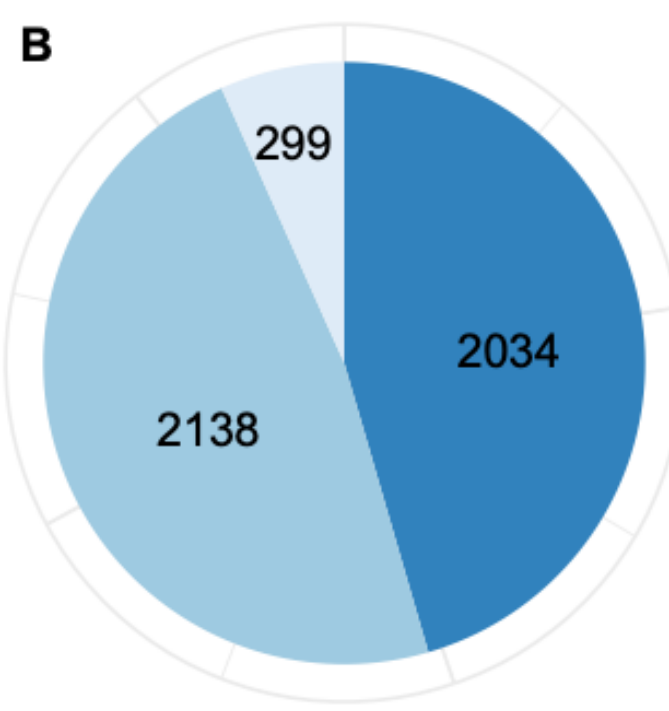

C

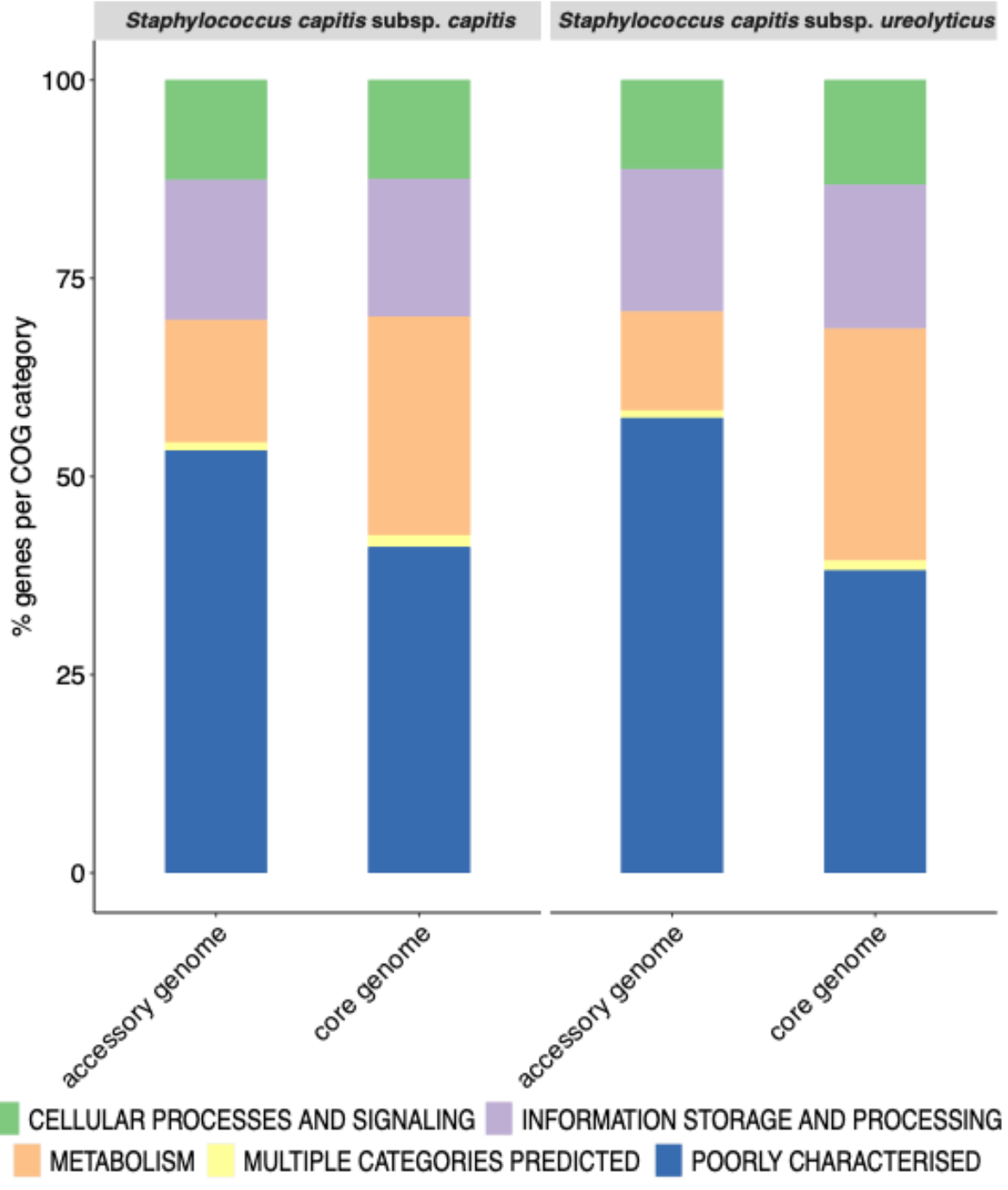

Figure 1 
bioRxiv preprint doi: https://doi.org/10.1101/2022.02.11.480064; this version posted February 11, 2022. The copyright holder for this preprint (which was not certified by peer review) is the author/funder, who has granted bioRxiv a license to display the preprint in perpetuity. It is made available under aCC-BY-NC-ND 4.0 International license.

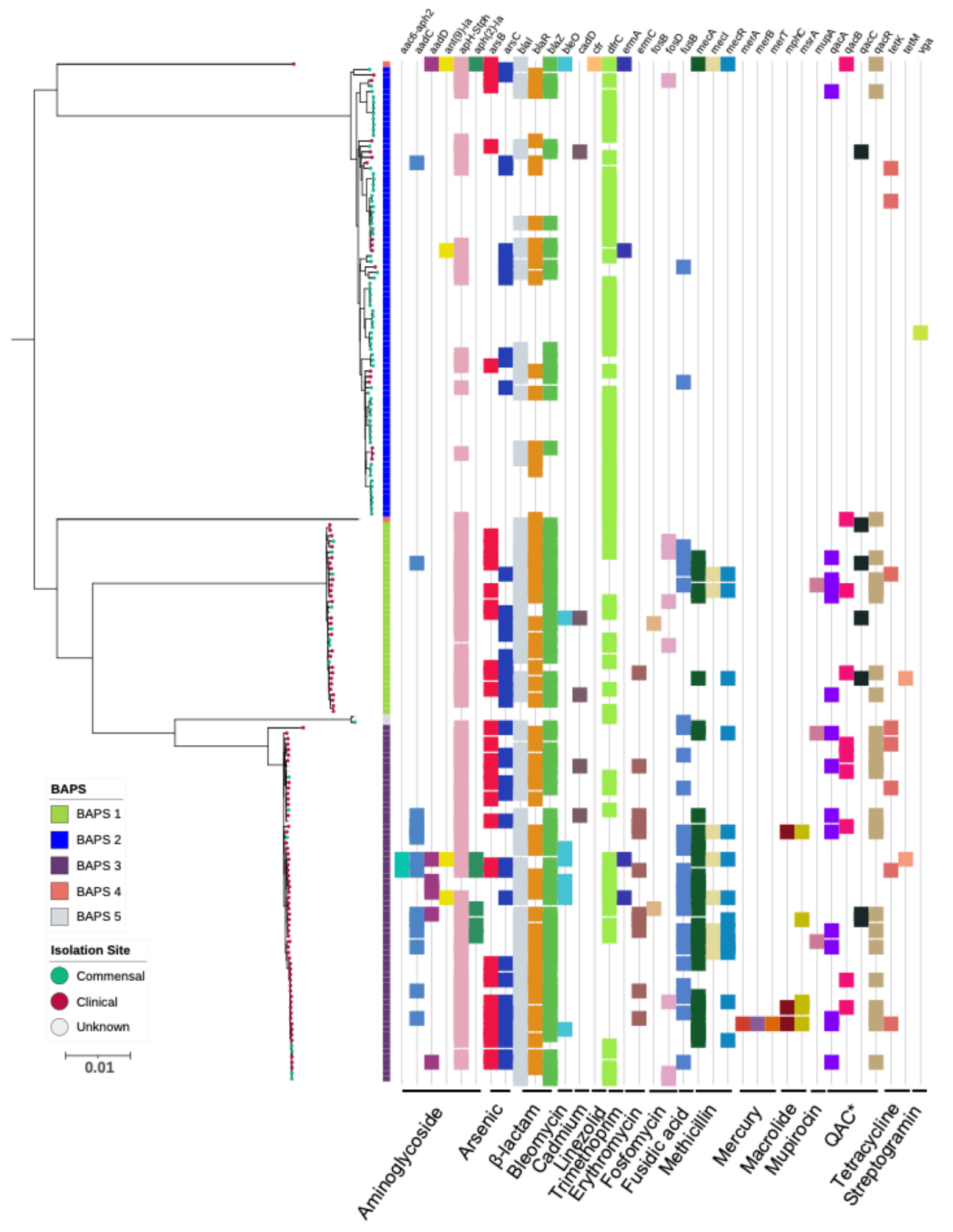


bioRxiv preprint doi: https://doi.org/10.1101/2022.02.11.480064; this version posted February 11, 2022. The copyright holder for this

preprint (which was not certified by peer review) is the author/funder, who has granted bioRxiv a license to display the preprint in perpetuity. It is made available under aCC-BY-NC-ND 4.0 International license.

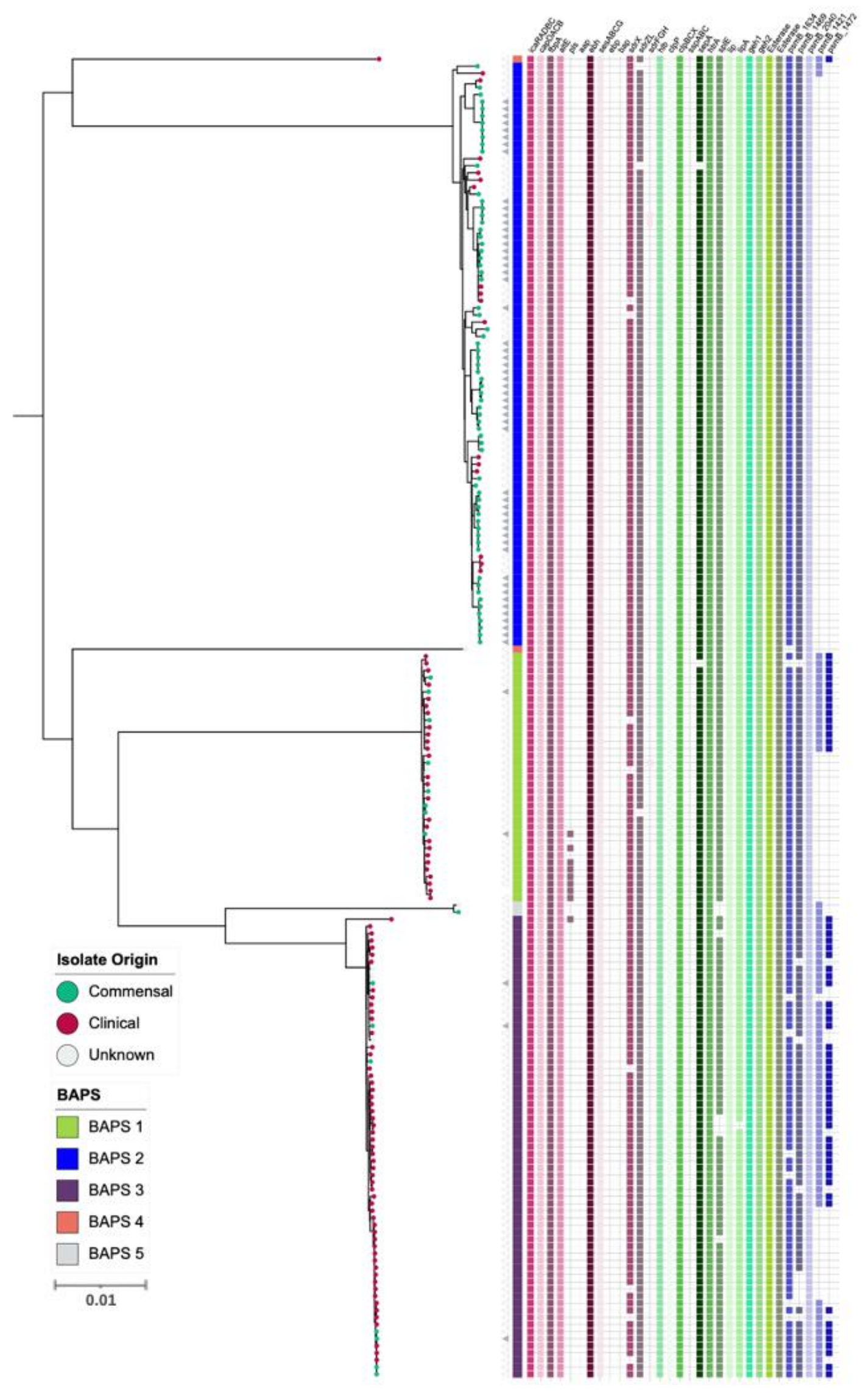


bioRxiv preprint doi: https://doi.org/10.1101/2022.02.11.480064; this version posted February 11, 2022. The copyright holder for this preprint (which was not certified by peer review) is the author/funder, who has granted bioRxiv a license to display the preprint in perpetuity. It is made available under aCC-BY-NC-ND 4.0 International license.

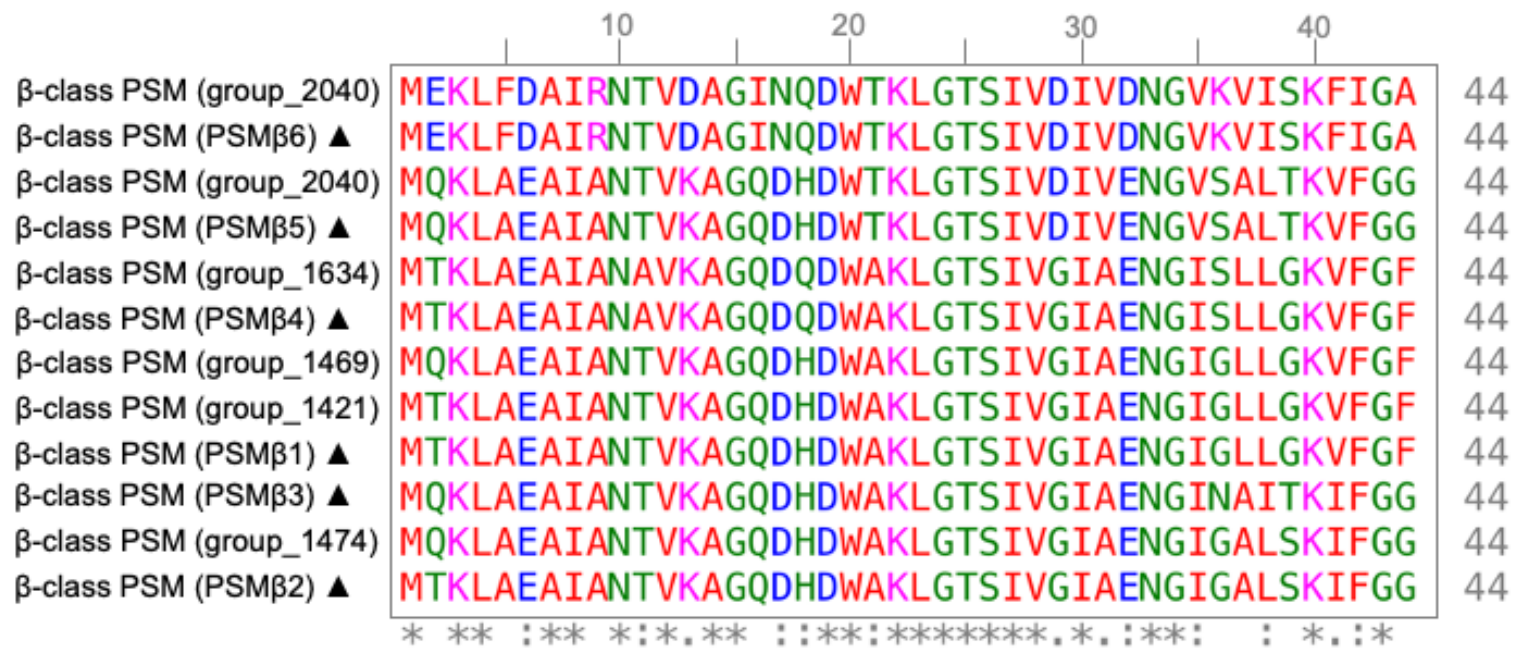


bioRxiv preprint doi: https://doi.org/10.1101/2022.02.11.480064; this version posted February 11, 2022. The copyright holder for this preprint (which was not certified by peer review) is the author/funder, who has granted bioRxiv a license to display the preprint in perpetuity. It is made available under aCC-BY-NC-ND 4.0 International license.

\begin{tabular}{|c|c|c|c|c|c|}
\hline Two-component system & S. aureus reference & S. capitis reference & $\begin{array}{c}\text { Presence in } S \text {. capitis } \\
\text { ssp. capitis (\%) }\end{array}$ & $\begin{array}{c}\text { Presence in S. capitis } \\
\text { ssp. ureolyticus }(\%)\end{array}$ & Function \\
\hline \multirow[t]{2}{*}{ walRK } & MW0018 & AYP1020_RS09955 & 100 & 100 & Cell wall maintenance, cell viability \\
\hline & MW0019 & AYP1020_RS09960 & 100 & 100 & \\
\hline \multirow[t]{2}{*}{ hptSR } & MW0198 & & 0 & 0 & Intracellular survival, uptake of hexose phosphate \\
\hline & MW0199 & & 0 & 0 & \\
\hline \multirow[t]{2}{*}{ lytSR } & MW0236 & & 0 & 0 & Autolysis, eDNA release, biofilm \\
\hline & MW0237 & & 0 & 0 & \\
\hline \multirow[t]{2}{*}{ graRS } & MW0621 & AYP1020_RS00130 & 100 & 100 & AMPs resistance, growth at low $\mathrm{pH}$ \\
\hline & MW0622 & AYP1020_RS00135 & 100 & 100 & \\
\hline \multirow[t]{2}{*}{ saeSR } & MW0667 & AYP1020_RS00365 & 100 & 100 & Virulence factors regulation \\
\hline & MW0668 & AYP1020_RS00360 & 100 & 100 & \\
\hline \multirow[t]{2}{*}{$\operatorname{tcs} 7 S R$} & MW1208 & AYP1020_RS03075 & 100 & 100 & Uncharacterised function \\
\hline & MW1209 & AYP1020_RS03080 & 100 & 100 & \\
\hline \multirow[t]{2}{*}{ arlRS } & MW1304 & AYP1020_RS03545 & 100 & 100 & Pathogenesis mechanisms \\
\hline & MW1305 & AYP1020_RS03540 & 100 & 100 & \\
\hline \multirow[t]{2}{*}{$\operatorname{sr} B A$} & MW1445 & AYP1020_RS03875 & 100 & 100 & Anaerobic respiration, metabolism, growth at low temperatures \\
\hline & MW1446 & AYP1020_RS03880 & 100 & 100 & \\
\hline \multirow[t]{2}{*}{ phoRP } & MW1636 & AYP1020_RS04760 & 100 & 100 & Phosphate uptake and homeostasis \\
\hline & MW1637 & AYP1020_RS04765 & 100 & 100 & \\
\hline \multirow[t]{2}{*}{ airRS } & MW1789 & AYP1020_RS05370 & 100 & 100 & Oxidative stress response \\
\hline & MW1790 & AYP1020_RS05375 & 100 & 100 & \\
\hline \multirow[t]{2}{*}{$\operatorname{var} R S$} & MW1824 & AYP1020_RS05700 & 100 & 100 & Cell wall-affecting antibiotic resistance, cell wall biosynthesis \\
\hline & MW1825 & AYP1020_RS05705 & 100 & 100 & \\
\hline \multirow[t]{2}{*}{$\operatorname{agr} C A$} & MW1962 & AYP1020_RS06010 & 100 & 100 & Quorom sensing control of adhesion and virulence factors \\
\hline & MW1963 & AYP1020_RS06005 & 100 & 100 & \\
\hline \multirow[t]{2}{*}{$k p d D E$} & MW2002 & AYP1020_RS09655 & 100 & 100 & Potassium homeostasis regulation \\
\hline & MW2003 & AYP1020_RS09660 & 100 & 100 & \\
\hline \multirow[t]{2}{*}{ hssRS } & MW2282 & AYP1020_RS07580 & 100 & 100 & Heme metabolism regulation \\
\hline & MW2283 & AYP1020_RS07585 & 100 & 100 & \\
\hline \multirow[t]{2}{*}{ nreCB } & MW2313 & AYP1020_RS07750 & 100 & 100 & Response to low oxygen, nitrate reduction \\
\hline & MW2314 & AYP1020_RS07755 & 100 & 100 & \\
\hline \multirow[t]{2}{*}{ braSR } & MW2544 & AYP1020_RS08920 & 100 & 100 & Antimicrobial peptide resistance \\
\hline & MW2545 & AYP1020_RS08925 & 100 & 100 & \\
\hline
\end{tabular}

Table 1 
bioRxiv preprint doi: https://doi.org/10.1101/2022.02.11.480064; this version posted February 11, 2022. The copyright holder for this

preprint (which was not certified by peer review) is the author/funder, who has granted bioRxiv a license to display the preprint in perpetuity. It is made available under aCC-BY-NC-ND 4.0 International license.

\begin{tabular}{|c|c|c|c|c|}
\hline Gene name & S. capitis reference & Annotation & S.capitis ssp. capitis (\%) & S.capitis ssp. ureolyticus (\%) \\
\hline group_2869 & & Hypothetical protein & 100 & 0 \\
\hline group_459 & & Putative phage head morphogenesis protein & 100 & 0 \\
\hline crtNX & & Dehydrosqualene desaturase & 100 & 4.76 \\
\hline hdcA & & Histidine decarboxylase proenzyme & 100 & 6.67 \\
\hline hsdM & & $\begin{array}{l}\text { Type I restriction-modification system methyltransferase } \\
\text { subunit }\end{array}$ & 100 & 21.90 \\
\hline group_1023 & & Putative transcriptional regulator & 100 & 29.52 \\
\hline group_2665 & AYP1020_RS09385 & Putative protein YjdF & 100 & 34.29 \\
\hline sasCX & & Cell-wall-anchored protein SasC & 100 & 35.24 \\
\hline $\operatorname{arcAX}$ & & Arginine deiminase & 98.72 & 6.67 \\
\hline $\operatorname{arcCX}$ & & Carbamate kinase $\mathrm{ArcC} 1$ & 98.72 & 7.62 \\
\hline $\operatorname{arcDX}$ & & $\begin{array}{l}\text { Arginine/ornithine APC family amino acid-polyamine- } \\
\text { organocation transporter antiporter }\end{array}$ & 98.72 & 7.62 \\
\hline $\operatorname{argFX}$ & & Ornithine carbamoyltransferase & 98.72 & 7.62 \\
\hline hsdSX & & $\begin{array}{l}\text { Type I restriction modification DNA specificity } \\
\text { protein;type I restriction modification system site } \\
\text { specificity determination subunit HsdS_1 }\end{array}$ & 98.72 & 7.62 \\
\hline trkG & & Trk family potassium $(\mathrm{K}+)$ transporter $\mathrm{ABC}$ protein & 98.72 & 11.43 \\
\hline $\operatorname{arsB}$ & & Arsenical pump membrane protein & 98.72 & 16.19 \\
\hline $\mathrm{cdr}$ & & Coenzyme A disulfide reductase & 98.72 & 19.05 \\
\hline $\operatorname{arsR}$ & & Arsenical resistance operon repressor & 98.72 & 19.05 \\
\hline group_2407 & & Arsenate reductase (glutaredoxin) & 98.72 & 20.95 \\
\hline arsA & & Arsenical pump-driving ATPase & 98.72 & 55.24 \\
\hline $\operatorname{arsD}$ & & Arsenical resistance operon trans-acting repressor ArsD & 92.31 & 37.14 \\
\hline pyrBX & & Aspartate carbamoyltransferase catalytic subunit & 98.72 & 0.95 \\
\hline group_1353 & AYP1020_RS02690 & Hypothetical protein & 0 & 100 \\
\hline group_798 & AYP1020_RS11575 & Hypothetical protein & 0 & 100 \\
\hline group_1413 & AYP1020_RS11570 & Hypothetical protein & 0 & 99.05 \\
\hline $\mathrm{icaCX}$ & AYP1020_RS08445 & $\begin{array}{l}\text { Putative poly-beta-16-N-acetyl-D-glucosamine export } \\
\text { protein }\end{array}$ & 0 & 98.10 \\
\hline fmrO & AYP1020_RS12300 & rRNA methyltransferase FmrO;hypothetical protein & 35.90 & 96.19 \\
\hline dapF & AYP1020_RS09635 & Diaminopimelate epimerase & 35.90 & 96.19 \\
\hline qorB & AYP1020_RS06480 & $\begin{array}{l}\text { Cobalt }(\mathrm{Co} 2+) \text { ABC superfamily ATP binding cassette } \\
\text { transporter membrane protein }\end{array}$ & 0 & 78.10 \\
\hline group_1472 & & Antibacterial protein (phenol soluble modulin) & 0 & 55.24 \\
\hline mqo & & Putative malate:quinone oxidoreductase 2 & 0 & 64.76 \\
\hline pfpI & & Intracellular protease & 0 & 60 \\
\hline opp1B & & $\begin{array}{l}\text { Oligopeptide ABC superfamily ATP binding cassette } \\
\text { transporter membrane protein }\end{array}$ & 35.90 & 84.76 \\
\hline opp1D & & $\begin{array}{l}\text { Oligopeptide } \mathrm{ABC} \text { superfamily ATP binding cassette } \\
\text { transporter } \mathrm{ABC} \text { protein }\end{array}$ & 35.90 & 84.76 \\
\hline group_687 & & Hypothetical protein & 35.90 & 84.76 \\
\hline group_585 & & MFS family major facilitator transporter & 35.90 & 84.76 \\
\hline opp1A & & $\begin{array}{l}\text { Oligopeptide ABC superfamily ATP binding cassette } \\
\text { transporter binding protein }\end{array}$ & 35.90 & 84.76 \\
\hline opp1C & & $\begin{array}{l}\text { Oligopeptide ABC superfamily ATP binding cassette } \\
\text { transporter membrane protein }\end{array}$ & 35.90 & 84.76 \\
\hline opp1F & & $\begin{array}{l}\text { ABC superfamily ATP binding cassette transporter } \mathrm{ABC} \\
\text { protein }\end{array}$ & 35.90 & 84.76 \\
\hline blaRX & & $\beta$-lactamase regulatory protein & 6.41 & 62.86 \\
\hline
\end{tabular}

Table 2 